\title{
On the Weak Solution to a Fractional Nonlinear Schrödinger Equation
}

\author{
Zujin Zhang, ${ }^{1}$ Xiaofeng Wang, ${ }^{2}$ and Zheng-an Yao ${ }^{3}$ \\ ${ }^{1}$ School of Mathematics and Computer Science, Gannan Normal University, Ganzhou 341000, China \\ ${ }^{2}$ College of Mathematics, Guangzhou University, Guangzhou 510006, China \\ ${ }^{3}$ Department of Mathematics, Sun Yat-sen University, Guangzhou 510275, China
}

Correspondence should be addressed to Zujin Zhang; zhangzujin361@163.com

Received 12 November 2013; Accepted 10 March 2014; Published 2 April 2014

Academic Editor: Elena Litsyn

Copyright (C) 2014 Zujin Zhang et al. This is an open access article distributed under the Creative Commons Attribution License, which permits unrestricted use, distribution, and reproduction in any medium, provided the original work is properly cited.

We obtain the existence of a global weak solution to a fractional nonlinear Schrödinger equation by the Galerkin method. Its uniqueness is also discussed. In our proof, we use harmonic analysis techniques and compactness arguments.

\section{Introduction}

This paper is concerned with the following fractional partial differential equations in the $n$ dimensional torus $\mathbb{T}^{n}$ :

$$
\frac{\partial u}{\partial t}-i \Lambda^{2 \alpha} u+|u|^{\rho} u=f
$$

where $\alpha \in(0,1)$ and $\rho \in(0, \infty)$ are real numbers and $i=$ $\sqrt{-1} . f(x, t)$ is given and $u(x, t)$ is a complex-valued function. Here, $\Lambda^{2 \alpha}=(-\Delta)^{\alpha}$, the fractional Laplacian with respect to $x$.

The fractional Laplacian operator appears in a wide class of physical systems and engineering problems, including Lévy flights, viscoelasticity, electrochemistry, control, porous media, electromagnetic, stochastic interfaces, and anomalous diffusion problems, and attracts the interests of many mathematicians; see [1-5], for example. The quasigeostrophic equation with fractional dissipation has been also extensively studied; see Constantin et al. [6-10], for example. In mathematical physics, the fractional Laplacian is often applied to describe many complicated phenomena via partial differential equations.

The Schrödinger equation is a fundamental equation in physics, which describes nonrelativistic quantum mechanical behavior. It is well known that Feynman and Hibbs [11] used path integrals over Brownian paths to derive the standard Schrödinger equation $(\alpha=1$ in (1)). Recently, Laskin [12, 13] showed that the path integral over the Lévy-like quantum mechanical paths allows us to generalize the classical quantum mechanics. Namely, if the path integral over Brownian trajectories leads to the well-known Schrödinger equation, then the path integral over Lévy trajectories leads to the fractional Schrödinger equation. The fractional Schrödinger equation includes the space derivative of order $2 \alpha$ instead of second-order space derivative in the standard Schrödinger equation. Laskin [14] showed the hermiticity of the fractional Hamilton operator and established the parity conservation law. Guo et al. [15] obtained the existence of a unique global smooth solution to the periodical boundary value problem for the fractional nonlinear Schrödinger equation.

Interestingly enough, there are also some other models involving the damping term $|u|^{\rho} u$; see $[16,17]$.

In studying (1), there exist some essential difficulties. First, since the fractional differential operator is defined by Fourier series and is nonlocal except when $\alpha \in \mathbb{N}$, which means that $\Lambda^{2 \alpha} u(x)$ depends not only on $u(y)$ for $y$ near $x$, but also on $u(y)$ for all $y$. Moreover, integration by part for nonlinear term is not valid. These bring new difficulties when doing energy estimate. And thus new harmonic analysis methods must be introduced to overcome these difficulties. Second, there are some difficulties in the convergence of the approximate solutions because of the nonlinear term. A compactness device should be given to treat this case. 
We now collect the notations in this paper. The fractional Laplacian $\Lambda^{2 \alpha} f$, for $\alpha \in \mathbb{R}$, can be defined as

$$
\widehat{\Lambda}^{2 \alpha} f_{k}=|k|^{2 \alpha} \widehat{f}_{k}, \quad k \in \mathbb{Z}^{n},
$$

where $\widehat{f}_{k}$ is the Fourier coefficients of $f$ :

$$
\widehat{f}_{k}=\frac{1}{(2 \pi)^{n}} \int_{\mathbb{T}^{n}} f(x) e^{-i k \cdot x} d x, \quad k \in \mathbb{Z}^{n} .
$$

We shall also invoke the notion of inhomogeneous Sobolev space $\dot{H}^{s}\left(\mathbb{T}^{n}\right) \quad(s \in \mathbb{R})$, which comprises all tempered distributions $f$ on $\mathbb{R}^{n}$ such that

$$
\|f\|_{H^{s}\left(\mathbb{T}^{n}\right)}=\left(\sum_{k \in \mathbb{Z}^{n} \backslash\{0\}}\left(1+|k|^{2}\right)^{s}\left|\widehat{u}_{k}\right|^{2}\right)^{1 / 2} .
$$

We now end this introduction by outlining the rest of this paper. In Section 2, we prove the existence of a weak solution to (1); see Theorem 8 . The uniqueness of such weak solutions is discussed in Section 3; see Theorem 9.

\section{Existence of a Weak Solution}

In this section, we prove the existence of a weak solution to the following system:

$$
\begin{gathered}
\frac{\partial u}{\partial t}-i \Lambda^{2 \alpha} u+|u|^{\rho} u=f, \quad(x, t) \in \mathbb{T}^{n} \times(0, T) \\
u(0)=u_{0}, \quad x \in \mathbb{T}^{n} .
\end{gathered}
$$

Let us first recall and prove some fundamental Lemmas.

Lemma 1 (see [18]). Let X be a Banach space; consider

$$
f \in L^{p}(0, T ; X), \quad \frac{\partial f}{\partial t} \in L^{p}(0, T ; X),
$$

for some $1 \leq p \leq \infty$; then $f$ is continuous map from $[0, T]$ to $X$.

Lemma 2 (see [18]). Let $\mathscr{D}$ be a bounded domain in $\mathbb{R}^{n} \times \mathbb{R}_{+}$, and $g_{k}, g$ are in $L^{q}(\mathscr{D})(1<q<\infty)$ with

$$
\left\|g_{k}\right\|_{L^{q}(\mathscr{D})} \leq C, \quad g_{k} \longrightarrow g \text {, a.e. } \mathscr{D} ;
$$

then $g_{k} \rightarrow g$ weakly in $L^{q}(\mathscr{D})$.

Lemma 3 (see [18]). Let $T>0,1<p_{1}, p_{2}<\infty$, and $B_{0}, B, B_{1}$ be three Banach spaces satisfying $B_{0} \hookrightarrow B \hookrightarrow B_{1}$, where $B_{0}, B_{1}$ are reflexive and the embedding $B_{0} \hookrightarrow B$ is compact. Endow the space

$$
W=\left\{w ; w \in L^{p_{0}}\left(0, T ; B_{0}\right), \frac{\partial w}{\partial t} \in L^{p_{1}}\left(0, T ; B_{1}\right)\right\}
$$

with the norm

$$
\|w\|_{W}=\|w\|_{L^{p_{0}}\left(0, T ; B_{0}\right)}+\left\|\frac{\partial w}{\partial t}\right\|_{L^{p_{1}}\left(0, T ; B_{1}\right)}, \quad \forall w \in W .
$$

Then the embedding $W \hookrightarrow L^{p_{0}}(0, T ; B)$ is compact.
In the following developments, we modify the methods in [19].

Proposition 4. Let $0<\alpha<1, x \in \mathbb{T}^{n}=\mathbb{R}^{n} / \mathbb{Z}^{n}$, and $u \in$ $\mathcal{S}\left(\mathbb{R}^{n}\right)$. Then

$$
\Lambda^{2 \alpha} u(x)=C_{\alpha} \sum_{\nu \in \mathbb{Z}^{n}} P V \int_{\mathbb{T}^{n}} \frac{u(x)-u(y)}{|x-y-\nu|^{n+2 \alpha}} \mathrm{d} y,
$$

with $C_{\alpha}>0$

Proof. First, we have

$$
\begin{aligned}
\Lambda^{2 \alpha} u(x) & =\sum_{|\nu|>0}|\nu|^{2 \alpha} \widehat{u}(\nu) e^{i v \cdot x} \\
& =-\sum_{|\nu|>0}|\nu|^{2 \alpha-2} \widehat{\Delta u}(\nu) e^{i v \cdot x} .
\end{aligned}
$$

Let $\Phi_{\varepsilon}(x)=\left(|x|^{2 \alpha-2}\right)_{\varepsilon} * \varphi_{\varepsilon}(x)$, where $\left(|x|^{2 \alpha-2}\right)_{\varepsilon}=|x|^{2 \alpha-2}$. $\chi(|x| / \varepsilon)$ with $\chi \in C^{\infty}([0, \infty))$,

$$
\chi(x)= \begin{cases}0, & \text { if }|x| \leq 1 \\ 1, & \text { if }|x| \geq 2\end{cases}
$$

and $\varphi_{\varepsilon}(x)=\varepsilon^{-n} \varphi(x / \varepsilon)$ is a standard approximation of identity, $0 \leq \varphi \in C^{\infty}\left(\mathbb{R}^{n}\right)$, supp $\varphi \subset B_{1}$, and $\int \varphi=1$. Now we can write

$$
\begin{aligned}
\Lambda^{2 \alpha} u(x) & =-\lim _{\varepsilon \rightarrow 0} \sum_{\nu \in \mathbb{Z}^{n}} \Phi_{\varepsilon}(\nu) \widehat{\Delta u}(\nu) e^{i v \cdot x} \\
& =-\lim _{\varepsilon \rightarrow 0}\left(\sum_{\nu \in \mathbb{Z}^{n}} \Phi_{\varepsilon}(\nu) e^{i v \cdot x}\right) *\left(\sum_{\nu \in \mathbb{Z}^{n}} \widehat{\Delta u}(\nu) e^{i v \cdot x}\right) .
\end{aligned}
$$

Poisson's summation then yields

$$
\begin{aligned}
\Lambda^{2 \alpha} u(x) & =-\lim _{\varepsilon \rightarrow 0}\left(\sum_{\nu \in \mathbb{Z}^{n}} \widehat{\Phi_{\varepsilon}}(x-v)\right) * \Delta u(x) \\
& =\lim _{\varepsilon \rightarrow 0} \sum_{\nu \in \mathbb{Z}^{n}} \int_{\mathbb{T}^{n}} \widehat{\Phi_{\varepsilon}}(x-y-v) \Delta[u(x)-u(y)] \mathrm{d} y \\
& =\lim _{\varepsilon \rightarrow 0} \sum_{\nu \in \mathbb{Z}^{n}} \int_{\mathbb{T}^{n}} \Delta\left(\widehat{\Phi_{\varepsilon}}\right)(x-y-v)[u(x)-u(y)] \mathrm{d} y .
\end{aligned}
$$

Due to the fact that

$$
\begin{gathered}
\widehat{\Phi_{\varepsilon}}(\eta)=\left(\widehat{|x|^{2 \alpha-2}}\right)_{\varepsilon} \cdot \widehat{\varphi_{\varepsilon}}(\eta)=\left(\widehat{|x|^{2 \alpha-2}}\right)_{\varepsilon} \cdot \widehat{\varphi}(\varepsilon \eta), \\
\Delta \widehat{\Phi_{\varepsilon}}(\eta)=\Delta\left(\left(\mid \widehat{\left.x\right|^{2 \alpha-2}}\right)_{\varepsilon}\right)(\eta) \cdot \widehat{\varphi}(\varepsilon \eta)+O(\varepsilon),
\end{gathered}
$$




$$
\begin{aligned}
\left(\widehat{|x|^{2 \alpha-2}}\right)_{\varepsilon}(y)= & \frac{c_{\alpha}}{|y|^{n+2 \alpha-2}} \\
& -\int_{\mathbb{T}^{n}} e^{-i y \cdot x}|x|^{2 \alpha-2}\left(1-\chi\left(\frac{|x|}{\varepsilon}\right)\right) \mathrm{d} x, \\
\Delta\left(\widehat{|x|^{2 \alpha-2}}\right)_{\varepsilon}(y)= & \frac{\tilde{c}_{\alpha}}{|y|^{n+2 \alpha}} \\
& -\int_{\mathbb{T}^{n}} e^{-i y \cdot x}|x|^{2 \alpha}\left(1-\chi\left(\frac{|x|}{\varepsilon}\right)\right) \mathrm{d} x,
\end{aligned}
$$

we obtain

$$
\begin{aligned}
\sum_{\nu \in \mathbb{Z}^{n}} \Delta\left(\widehat{\Phi_{\varepsilon}}\right)(y-\nu)= & \widetilde{c}_{\alpha} \sum_{\nu \in \mathbb{Z}^{n}} \frac{1}{|y-\nu|^{n+2 \alpha}} \\
& +O\left(\sum_{\nu \in \mathbb{Z}^{n}} \frac{1}{|y-\nu|^{n+\delta}} O\left(\varepsilon^{\delta}\right)\right),
\end{aligned}
$$

for some $\delta>0$.

Therefore,

$$
\begin{aligned}
& \Lambda^{2 \alpha} u(x) \\
& \quad=\lim _{\varepsilon \rightarrow 0} \sum_{\nu \in \mathbb{Z}^{n}} \int_{\mathbb{T}^{n}} \Delta\left(\widehat{\Phi_{\varepsilon}}\right)(x-y-v)[u(x)-u(y)] \mathrm{d} y \\
& \quad=C_{\alpha} \sum_{\nu \in \mathbb{Z}^{n}} P V \int_{\mathbb{V}^{n}} \frac{u(x)-u(y)}{|x-y-\nu|^{n+2 \alpha}} \mathrm{d} y .
\end{aligned}
$$

Lemma 5. Let $\mathbb{T}^{n}=\mathbb{R}^{n} / \mathbb{Z}^{n}$, and let $u$ be a complex-valued function satisfying $u, u^{2 \alpha} \in L^{p}\left(\mathbb{R}^{n}\right)$ with $0<\alpha<1$ and $1 \leq$ $p<\infty$. Then

$$
\operatorname{Re}\left(\int_{\mathbb{T}^{n}}|u|^{p-2} u \overline{\Lambda^{2 \alpha} u} \mathrm{~d} x\right) \geq 0 .
$$

Proof. For $0<\alpha<1$, by invoking Proposition 4 and changing of variables, we have

$$
\begin{aligned}
& \operatorname{Re}\left(\int_{\mathbb{T}^{n}}|u|^{p-2} u \overline{\Lambda^{2 \alpha} u} \mathrm{~d} x\right) \\
& =C_{\alpha} \lim _{\varepsilon \rightarrow 0} \operatorname{Re}\left(\iint_{|y-x| \geq \varepsilon}|u(x)|^{p-2} u(x) \frac{\overline{u(x)-u(y)}}{|x-y|^{n+2 \alpha}} \mathrm{d} y \mathrm{~d} x\right) \\
& =-C_{\alpha} \lim _{\varepsilon \rightarrow 0} \operatorname{Re}\left(\int_{|y-x| \geq \varepsilon}|u(y)|^{p-2} u(y) \frac{\overline{u(x)-u(y)}}{|x-y|^{n+2 \alpha}} \mathrm{d} y \mathrm{~d} x\right) \\
& =\frac{C_{\alpha}}{2} \lim _{\varepsilon \rightarrow 0} \operatorname{Re}\left(\iint_{|y-x| \geq \varepsilon} \frac{\left[|u(x)|^{p-2} u(x)-|u(y)|^{p-2} u(y)\right]}{\left.\times \frac{u(x)-u(y)}{|x-y|^{n+2 \alpha}} \mathrm{d} y \mathrm{~d} x\right)}\right.
\end{aligned}
$$

$\geq 0$.
In the following Lemma, we give a characterization of the eigenvalues and eigenvectors of the pseudodifferential operators $\Lambda^{2 \alpha}$.

Lemma 6. Let $\mathbb{T}^{n}=\mathbb{R}^{n} / \mathbb{Z}^{n}$. Then there exist a sequence of real numbers $\left\{\lambda_{j}\right\}_{j=1}^{\infty}$ and a sequence of periodical functions $\left\{\omega_{j}\right\}_{j=1}^{\infty} \subset H^{2 \alpha}\left(\mathbb{T}^{n}\right)$, such that

$$
\Lambda^{2 \alpha} \omega_{j}=\lambda_{j} \omega_{j}, \quad j=1,2,3, \ldots
$$

Moreover, $\left\{\omega_{j}\right\}_{j=1}^{\infty}$ is a basis of $H^{2 \alpha}\left(\mathbb{T}^{n}\right)$ satisfying

$$
\begin{gathered}
\left(\omega_{j}, \omega_{k}\right) \equiv \int_{\mathbb{T}^{n}} \omega_{j} \overline{\omega_{k}} \mathrm{~d} x=\delta_{j k} \\
\left(\Lambda^{2 \alpha} \omega_{j}, \omega_{k}\right)=\lambda_{j} \delta_{j k}
\end{gathered}
$$

for $j, k=1,2,3, \ldots$

Notice that the lemma is a direct consequence of elliptic regularity and functional analysis; see [20], for example.

Let us now give the weak formulation of (5)-(6).

Definition 7. Let $T>0, f \in L^{2}\left(0, T ; H^{\alpha}\left(\mathbb{T}^{n}\right)\right), \partial f / \partial t \in$ $L^{2}\left(0, T ; L^{2}\left(\mathbb{T}^{n}\right)\right)$, and $u_{0} \in H^{\alpha}\left(\mathbb{T}^{n}\right) \cap L^{2(\rho+1)}\left(\mathbb{T}^{n}\right)$. A measurable complex-valued function $u(x, t)$ is said to be a weak solution to $(5)-(6)$ on $[0, T]$, provided the following:

(1) $u \in L^{\infty}\left(0, T ; H^{\alpha}\left(\mathbb{T}^{n}\right)\right) \cap L^{p}\left(0, T ; L^{p}\left(\mathbb{T}^{n}\right)\right)$ and $\partial u / \partial t \epsilon$ $L^{\infty}\left(0, T ; L^{2}\left(\mathbb{T}^{n}\right)\right)$;

(2) $u$ satisfies (5) in the sense of distributions;

(3) $u(x, 0)=u_{0}(x)$ a.e. $\mathbb{T}^{n}$.

Here and hereafter, $p=\rho+2$.

Now, we state our existence results in the following theorem.

Theorem 8. Let $T>0$ be a given time, and

$$
\begin{gathered}
u_{0} \in H^{\alpha}\left(\mathbb{T}^{n}\right) \cap L^{2(\rho+1)}\left(\mathbb{T}^{n}\right), \\
f \in L^{2}\left(0, T ; H^{\alpha}\left(\mathbb{T}^{n}\right)\right), \quad \frac{\partial f}{\partial t} \in L^{2}\left(0, T ; L^{2}\left(\mathbb{T}^{n}\right)\right) .
\end{gathered}
$$

Then there exists at least one weak solution $u$ to (5)-(6) on $[0, T]$, taking $u_{0}$ as initial data.

Proof. We use Galerkin method.

Step 1. Construction of approximate solution.

Let $\left\{\omega_{j}\right\}_{j=1}^{\infty}$ be given as in Lemma 6. We consider the approximate solution which has the form

$$
u_{m}(t)=\sum_{j=1}^{m} c_{j m}(t) \omega_{j},
$$


where $c_{j m}$ satisfy the following ordinary differential system:

$$
\begin{gathered}
\left(\frac{\partial u_{m}}{\partial t}(t), \omega_{j}\right)-i\left(\Lambda^{2 \alpha} u_{m}(t), \omega_{j}\right) \\
+\left(\left|u_{m}(t)\right|^{\rho} u_{m}(t), \omega_{j}\right) \\
=\left(f(t), \omega_{j}\right), \quad 1 \leq j \leq m, \\
u_{m}(0)=u_{0 m}=\sum_{j=1}^{m} \alpha_{j m} \omega_{j} \longrightarrow u_{0} \quad \text { in } H^{\alpha}\left(\mathbb{T}^{n}\right) \cap L^{2(\rho+1)}\left(\mathbb{T}^{n}\right), \\
\text { as } m \longrightarrow \infty .
\end{gathered}
$$

Here and hereafter, $(u, v)=\int_{\mathbb{T}^{n}} u \bar{v} \mathrm{~d} x$ is the inner product in $L^{2}\left(\mathbb{T}^{n}\right)$.

The system (26)-(27) is nonsingular because $\left\{\omega_{j}\right\}$ are linear independent. Thus we may apply standard theory of ordinary differential equations to obtain the existence of a local solution to equations (26)-(27) on $\left[0, t_{m}\right]$, for some $t_{m}>$ 0 . We shall then, in the next step, establish some a priori estimates of the obtained solutions. This will ensure that $t_{m}=$ $T$.

Step 2. A priori estimates.

By multiplying (26) by $\overline{c_{j m}}(t)$ and summing with $1 \leq j \leq$ $m$, we have

$$
\begin{aligned}
& \left(\frac{\partial u_{m}}{\partial t}(t), u_{m}(t)\right)+i\left(\Lambda^{\alpha} u_{m}(t), \Lambda^{\alpha} u_{m}(t)\right) \\
& \quad+\left(\left|u_{m}(t)\right|^{\rho} u_{m}(t), u_{m}(t)\right)=\left(f(t), u_{m}(t)\right) .
\end{aligned}
$$

Taking real part of (28) and invoking Hölder inequality then yields

$$
\begin{aligned}
\frac{1}{2} \frac{\mathrm{d}}{\mathrm{d} t} & \left\|u_{m}(t)\right\|_{L^{2}\left(\mathbb{T}^{n}\right)}^{2}+\int_{\mathbb{T}^{n}}\left|u_{m}(t)\right|^{p} \mathrm{~d} x \\
& =\operatorname{Re}\left(f(t), u_{m}(t)\right) \\
& \leq\left\|u_{m}(t)\right\|_{L^{2}\left(\mathbb{T}^{n}\right)}^{2}+\|f(t)\|_{L^{2}\left(\mathbb{T}^{n}\right)}^{2} .
\end{aligned}
$$

By Gronwall's inequality, we have $u_{m}$ is bounded in $L^{\infty}\left(0, T ; L^{2}\left(\mathbb{T}^{n}\right)\right), \quad L^{p}\left(0, T ; L^{p}\left(\mathbb{T}^{n}\right)\right)$.

By multiplying (26) by $\lambda_{j} \overline{c_{j m}}(t)$, summing with $1 \leq j \leq m$, and noticing (23), we get

$$
\begin{aligned}
& \left(\frac{\partial \Lambda^{\alpha} u_{m}}{\partial t}(t), \Lambda^{\alpha} u_{m}\right)-i\left\|\Lambda^{2 \alpha} u_{m}(t)\right\|_{L^{2}\left(\mathbb{T}^{n}\right)}^{2} \\
& \quad+\left(\left|u_{m}(t)\right|^{\rho} u_{m}(t), \Lambda^{2 \alpha} u_{m}(t)\right)=\left(\Lambda^{\alpha} f(t), \Lambda^{\alpha} u_{m}(t)\right) .
\end{aligned}
$$

Thanks to Lemma 5, we may consider the real part of (31) to obtain

$$
\frac{1}{2} \frac{\mathrm{d}}{\mathrm{d} t}\left\|\Lambda^{\alpha} u_{m}\right\|_{L^{2}\left(\mathbb{T}^{n}\right)} \leq \operatorname{Re}\left(\Lambda^{\alpha} f(t), \Lambda^{\alpha} u_{m}\right)
$$

By Gronwall's inequality again, we have

$$
u_{m} \text { is bounded in } L^{\infty}\left(0, T ; H^{\alpha}\left(\mathbb{T}^{n}\right)\right) .
$$

By multiplying (26) by $\overline{c_{j m}^{\prime}}(t)$, summing with $1 \leq j \leq m$, and taking $t=0$, we have

$$
\begin{aligned}
& \left\|\frac{\partial u_{m}}{\partial t}(0)\right\|_{L^{2}\left(\mathbb{T}^{n}\right)}^{2}-\left.\frac{i}{2} \frac{\mathrm{d}}{\mathrm{d} t}\right|_{t=0}\left\|\Lambda^{\alpha} u_{m}(t)\right\|_{L^{2}\left(\mathbb{T}^{n}\right)} \\
& \quad+\left(\left|u_{m}(0)\right|^{\rho} u_{m}(0), \frac{\partial u_{m}}{\partial t}(0)\right)=\left(f(0), \frac{\partial u_{m}}{\partial t}(0)\right) .
\end{aligned}
$$

Looking into the real part of (34), we see

$$
\begin{aligned}
& \left\|\frac{\partial u_{m}}{\partial t}(0)\right\|_{L^{2}\left(\mathbb{T}^{n}\right)}^{2} \\
& \quad \leq\left(\left\|u_{m}(0)\right\|_{L^{2(\rho+1)}\left(\mathbb{T}^{n}\right)}+\|f(0)\|_{L^{2}\left(\mathbb{T}^{n}\right)}\right)\left\|\frac{\partial u_{m}}{\partial t}(0)\right\|_{L^{2}\left(\mathbb{T}^{n}\right)} .
\end{aligned}
$$

Thus,

$$
\left\|\frac{\partial u_{m}}{\partial t}(0)\right\|_{L^{2}\left(\mathbb{T}^{n}\right)} \leq C .
$$

Now we can obtain the estimate for $u_{m}^{\prime}(t)$. By differentiating (26) with respect to $t$, we get

$$
\begin{gathered}
\left(\frac{\partial^{2} u_{m}}{\partial t^{2}}(t), \omega_{j}\right)+i\left(\frac{\partial \Lambda^{2 \alpha} u_{m}}{\partial t}(t), \omega_{j}\right) \\
+\left(\frac{\partial}{\partial t}\left(\left|u_{m}(t)\right|^{\rho} u_{m}(t)\right), \omega_{j}\right) \\
=\left(\frac{\partial f}{\partial t}(t), \omega_{j}\right), \quad 1 \leq j \leq m .
\end{gathered}
$$

By multiplying (37) by $\overline{c_{j m}^{\prime}}(t)$ and summing with $1 \leq j \leq m$, we have

$$
\begin{gathered}
\frac{1}{2} \frac{\mathrm{d}}{\mathrm{d} t}\left\|\frac{\partial u_{m}}{\partial t}(t)\right\|_{L^{2}\left(\mathbb{T}^{n}\right)}^{2}-\frac{i}{2}\left\|\frac{\partial \Lambda^{\alpha} u_{m}}{\partial t}(t)\right\|_{L^{2}\left(\mathbb{T}^{n}\right)} \\
\quad+\left(\frac{\partial}{\partial t}\left(\left|u_{m}(t)\right|^{\rho} u_{m}(t)\right), \frac{\partial u_{m}}{\partial t}(t)\right)=\left(\frac{\partial f}{\partial t}(t), \frac{\partial u_{m}}{\partial t}(t)\right) .
\end{gathered}
$$

Notice that

$$
\begin{aligned}
\left(\frac{\partial}{\partial t}\left(\left|u_{m}(t)\right|^{\rho} u_{m}(t)\right), \frac{\partial u_{m}}{\partial t}(t)\right) & \\
= & \int_{\mathbb{T}^{n}}\left|u_{m}(t)\right|^{\rho}\left|\frac{\partial u_{m}}{\partial t}(t)\right|^{2} \mathrm{~d} x \\
& +\frac{\rho}{2} \int_{\mathbb{T}^{n}}\left|u_{m}(t)\right|^{\rho-2} \\
& \times\left(\left|u_{m}(t) \frac{\partial \overline{u_{m}}}{\partial t}(t)\right|^{2}+\left|u_{m}(t)\right|^{2}\left|\frac{\partial u_{m}}{\partial t}\right|^{2}\right) \mathrm{d} x
\end{aligned}
$$

$\geq 0$. 
Taking the real part of (38) then yields

$$
\begin{gathered}
\frac{\mathrm{d}}{\mathrm{d} t}\left\|\frac{\partial u_{m}}{\partial t}(t)\right\|_{L^{2}\left(\mathbb{T}^{n}\right)}^{2} \\
\leq 2 \operatorname{Re}\left(\frac{\partial f}{\partial t}(t), \frac{\partial u_{m}}{\partial t}(t)\right) \\
\leq\left\|\frac{\partial f}{\partial t}(t)\right\|_{L^{2}\left(\mathbb{T}^{n}\right)}^{2}+\left\|\frac{\partial u_{m}}{\partial t}(t)\right\|_{L^{2}\left(\mathbb{T}^{n}\right)}^{2} .
\end{gathered}
$$

By Gronwall's inequality, we deduce

$$
\frac{\partial u_{m}}{\partial t} \text { is bounded in } L^{\infty}\left(0, T ; L^{2}\left(\mathbb{T}^{n}\right)\right) .
$$

Step 3. Convergence process.

By (30), (33), and (41), we have, up to a subsequence, still denoted by $u_{m}$, that

$$
\begin{aligned}
& u_{m} \rightarrow u \text { weakly } * \text { in } L^{\infty}\left(0, T ; H^{\alpha}\left(\mathbb{T}^{n}\right)\right), \quad \text { as } m \longrightarrow \infty, \\
& u_{m} \rightarrow u \text { weakly in } L^{p}\left(0, T ; L^{p}\left(\mathbb{T}^{n}\right)\right), \quad \text { as } m \longrightarrow \infty,(43) \\
& \frac{\partial u_{m}}{\partial t} \rightarrow \frac{\partial u}{\partial t} \text { weakly in } L^{\infty}\left(0, T ; L^{2}\left(\mathbb{T}^{n}\right)\right), \quad \text { as } m \longrightarrow \infty .
\end{aligned}
$$

Thus by Lemma 3, we find

$$
\begin{array}{r}
u_{m} \longrightarrow u \text { strongly in } L^{\infty}\left(0, T ; L^{2}\left(\mathbb{T}^{n}\right)\right), \text { a.e. } \mathbb{T}^{n}, \\
\text { as } m \longrightarrow \infty .
\end{array}
$$

By (44), there exists a function $w=w(x, t)$ such that

$$
\left|u_{m}\right|^{\rho} u_{m} \rightarrow w(x, t) \text { weakly in } L^{q}\left(0, T ; L^{q}\left(\mathbb{T}^{n}\right)\right),
$$

$$
\text { as } m \longrightarrow \infty \text {, }
$$

with $(1 / p)+(1 / q)=1$. By Lemma 2 and the fact that

$$
\left|u_{m}\right|^{\rho} u_{m} \longrightarrow|u|^{\rho} u \text {, a.e. } \mathbb{T}^{n},
$$

we see

$$
w=|u|^{\rho} u .
$$

Fix $j$; we now pass to limit $m \rightarrow \infty$ in (26) to deduce

$$
\begin{gathered}
\left(\frac{\partial u}{\partial t}(t), \omega_{j}\right)-i\left(\Lambda^{\alpha} u(t), \Lambda^{\alpha} \omega_{j}\right)+\left(|u(t)|^{\rho} u(t), \omega_{j}\right) \\
=\left(f(t), \omega_{j}\right) .
\end{gathered}
$$

A simple density argument then shows

$$
\begin{aligned}
& \left(\frac{\partial u}{\partial t}(t), v\right)+i\left(\Lambda^{\alpha} u(t), \Lambda^{\alpha} v\right)+\left(|u(t)|^{\rho} u(t), v\right) \\
& =(f(t), v)
\end{aligned}
$$

for all $v \in H^{\alpha}\left(\mathbb{T}^{n}\right) \cap L^{p}\left(\mathbb{T}^{n}\right)$.

The proof of Theorem 8 is completed.

\section{Uniqueness of Weak Solutions}

In this section, we will discuss the uniqueness of weak solutions of (5)-(6). More precisely, we have the following theorem.

Theorem 9. Assume as in Theorem 8. Then there exists a unique weak solution of (5)-(6).

Proof. Let $u$ and $v$ be two weak solutions of (5)-(6), given in Theorem 8 , with the same datum. Then $w=u-v$ satisfies

$$
\begin{gathered}
\frac{\partial w}{\partial t}+i \Lambda^{2 \alpha} w=|v|^{\rho} v-|u|^{\rho} u, \\
w(0)=0 .
\end{gathered}
$$

Also, we have

$$
\begin{gathered}
w \in L^{\infty}\left(0, T ; H^{\alpha}\left(\mathbb{T}^{n}\right)\right) \cap L^{p}\left(0, T ; L^{p}\left(\mathbb{T}^{n}\right)\right), \\
\frac{\partial w}{\partial t} \in L^{\infty}\left(0, T ; L^{2}\left(\mathbb{T}^{n}\right)\right) .
\end{gathered}
$$
obtain

By taking the inner product of (51) with $w$ in $L^{2}\left(\mathbb{T}^{n}\right)$, we

$$
\begin{aligned}
& \frac{1}{2} \frac{\mathrm{d}}{\mathrm{d} t}\|w\|_{L^{2}\left(\mathbb{T}^{n}\right)}^{2}-i\|w\|_{H^{\alpha}\left(\mathbb{T}^{n}\right)}^{2} \\
&=\int_{\mathbb{T}^{n}}\left(|v|^{\rho} v-|u|^{\rho} u\right) \overline{(u-v)} \mathrm{d} x .
\end{aligned}
$$

By taking the real part of (54) and noticing

$$
\operatorname{Re}\left(\int_{\mathbb{T}^{n}}\left(|v|^{\rho} v-|u|^{\rho} u\right) \overline{(u-v)} \mathrm{d} x\right) \leq 0,
$$

we have

$$
\|w\|_{L^{2}\left(\mathbb{T}^{n}\right)}^{2} \leq\|w(0)\|_{L^{2}\left(\mathbb{T}^{n}\right)}^{2}=0 .
$$

Thus $w=0$ and $u \equiv v$ a.e. in $\mathbb{T}^{n}$.

\section{Conflict of Interests}

The authors declare that there is no conflict of interests regarding the publication of this paper.

\section{Acknowledgments}

Zujin Zhang was partially supported by the Youth Natural Science Foundation of Jiangxi Province (20132BAB211007 and 20122BAB201014) and the National Natural Science Foundation of China (11326138 and 11361004). Xiaofeng Wang was partially supported by the Guangzhou higher Education Science and Technology Projection (2012A018). Zheng-an Yao was partially supported by the National Natural Science Foundation of China (11271381).

\section{References}

[1] P. Li and Z. Zhai, "Well-posedness and regularity of generalized Navier-Stokes equations in some critical-spaces," Journal of Functional Analysis, vol. 259, no. 10, pp. 2457-2519, 2010. 
[2] K. S. Miller and B. Ross, An Introduction to the Fractional Calculus and Fractional Differential Equations, John Wiley \& Sons, New York, NY, USA, 1993.

[3] I. Podlubny, Fractional Differential Equations, vol. 198 of Mathematics in Science and Engineering, Academic Press, San Diego, Calif, USA, 1999.

[4] J. Shatah and C. Zeng, "Schrödinger maps and antiferromagnetic chains," Communications in Mathematical Physics, vol. 262, no. 2, pp. 299-315, 2006.

[5] P.-L. Sulem, C. Sulem, and C. Bardos, "On the continuous limit for a system of classical spins," Communications in Mathematical Physics, vol. 107, no. 3, pp. 431-454, 1986.

[6] P. Constantin, "Energy spectrum of quasigeostrophic turbulence," Physical Review Letters, vol. 89, no. 18, Article ID 184501, 4 pages, 2002.

[7] P. Constantin, "On the Euler equations of incompressible fluids," Bulletin of the American Mathematical Society, vol. 44, no. 4, pp. 603-621, 2007.

[8] P. Constantin, D. Cordoba, and J. Wu, "On the critical dissipative quasi-geostrophic equation," Indiana University Mathematics Journal, vol. 50, pp. 97-107, 2001.

[9] P. Constantin, A. J. Majda, and E. Tabak, "Formation of strong fronts in the Q-D quasigeostrophic thermal active scalar," Nonlinearity, vol. 7, no. 6, pp. 1495-1533, 1994.

[10] P. Constantin and J. Wu, "Behavior of solutions of 2D quasigeostrophic equations," SIAM Journal on Mathematical Analysis, vol. 30, no. 5, pp. 937-948, 1999.

[11] R. P. Feynman and A. R. Hibbs, Quantum Mechanics and Path Integrals, McGraw-Hill, New York, NY, USA, 1965.

[12] N. Laskin, "Fractional quantum mechanics and Lévy path integrals," Physics Letters A, vol. 268, no. 4-6, pp. 298-305, 2000.

[13] N. Laskin, "Fractional quantum mechanics," Physical Review E, vol. 62, pp. 3135-3145, 2000.

[14] N. Laskin, "Fractional Schrödinger equation," Physical Review E, vol. 66, no. 5, Article ID 056108, 7 pages, 2002.

[15] B. Guo, Y. Han, and J. Xin, "Existence of the global smooth solution to the period boundary value problem of fractional nonlinear Schrödinger equation," Applied Mathematics and Computation, vol. 204, no. 1, pp. 468-477, 2008.

[16] Z. Zhang, X. Wu, and M. Lu, "On the uniqueness of strong solution to the incompressible Navier-Stokes equations with damping," Journal of Mathematical Analysis and Applications, vol. 377, no. 1, pp. 414-419, 2011.

[17] Z. J. Zhang, X. F. Wang, and Z.-A. Yao, "On a fractional nonlinear hyperbolic equation arising from relative theory," Abstract and Applied Analysis, vol. 2013, Article ID 548562, 6 pages, 2013.

[18] J. L. Lions and Quelques, Méthodes de Résolution des Probléms aux Limites Non Linéaire, Sun Yat-sen University Press, GuangZhou, China, Chinese edition, 1996.

[19] A. Córdoba and D. Córdoba, "A maximum principle applied to quasi-geostrophic equations," Communications in Mathematical Physics, vol. 249, no. 3, pp. 511-528, 2004.

[20] R. Temam, Infinite-Dimensional Dynamical Systems in Mechanics and Physics, vol. 68 of Applied Mathematical Sciences, Springer, New York, NY, USA, 1988. 


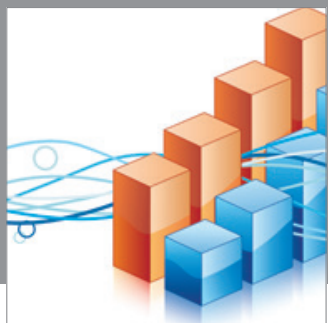

Advances in

Operations Research

mansans

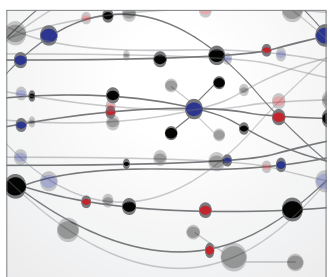

The Scientific World Journal
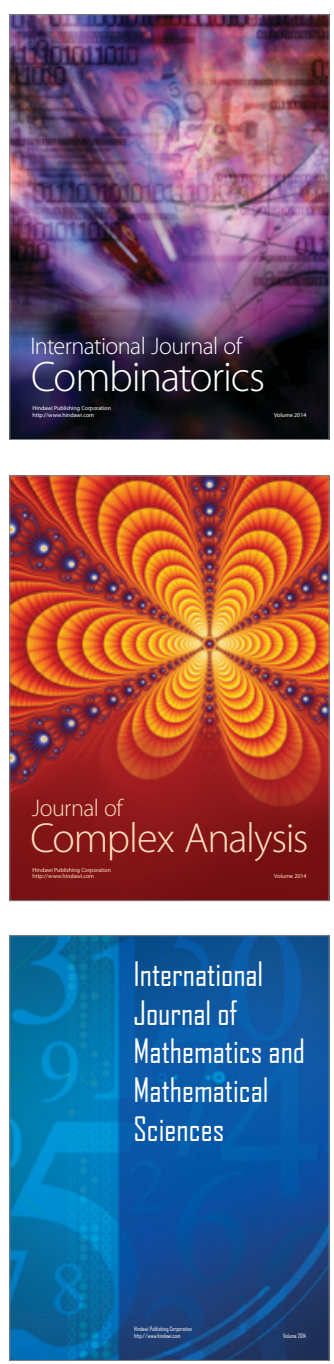
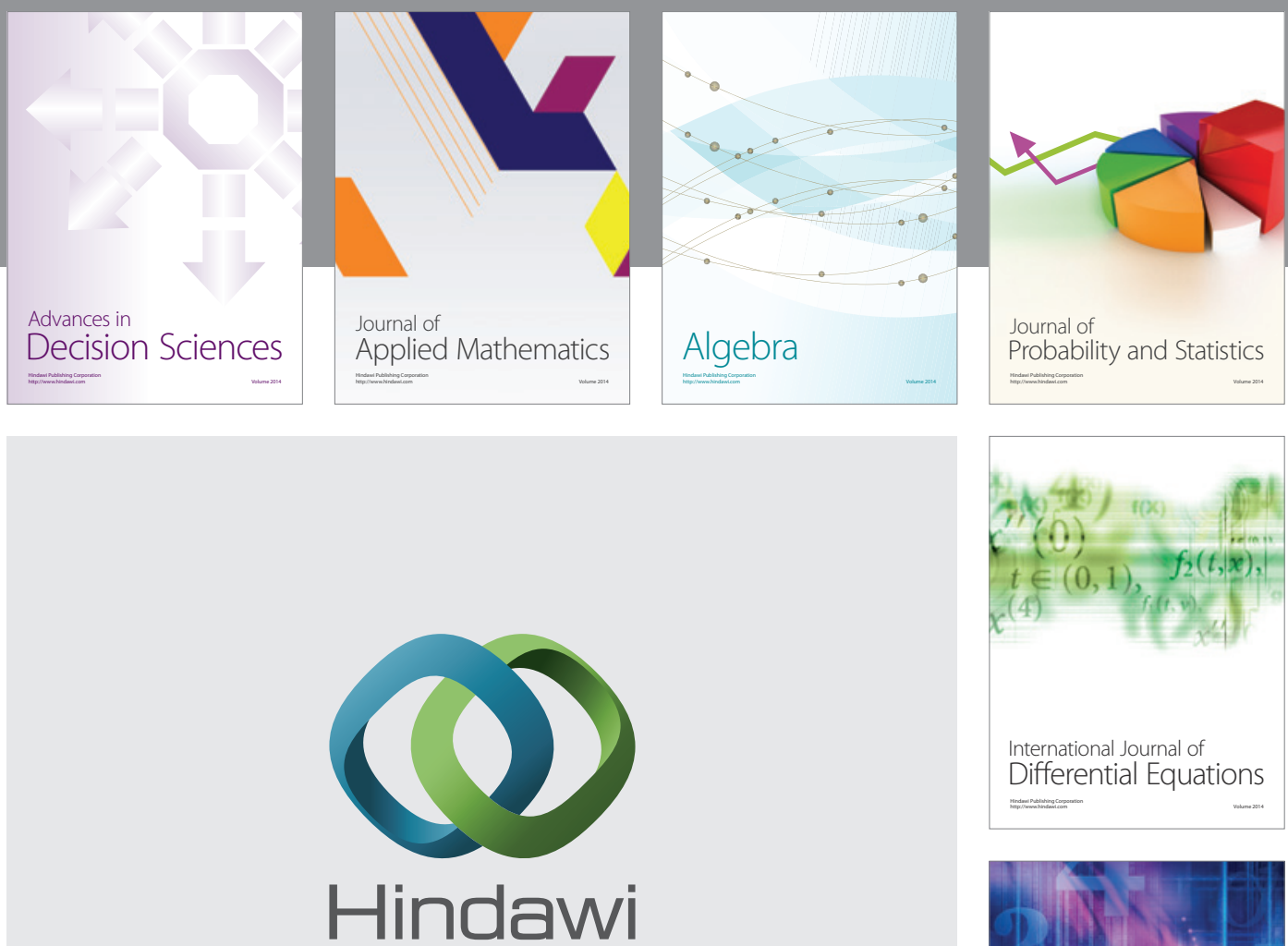

Submit your manuscripts at http://www.hindawi.com
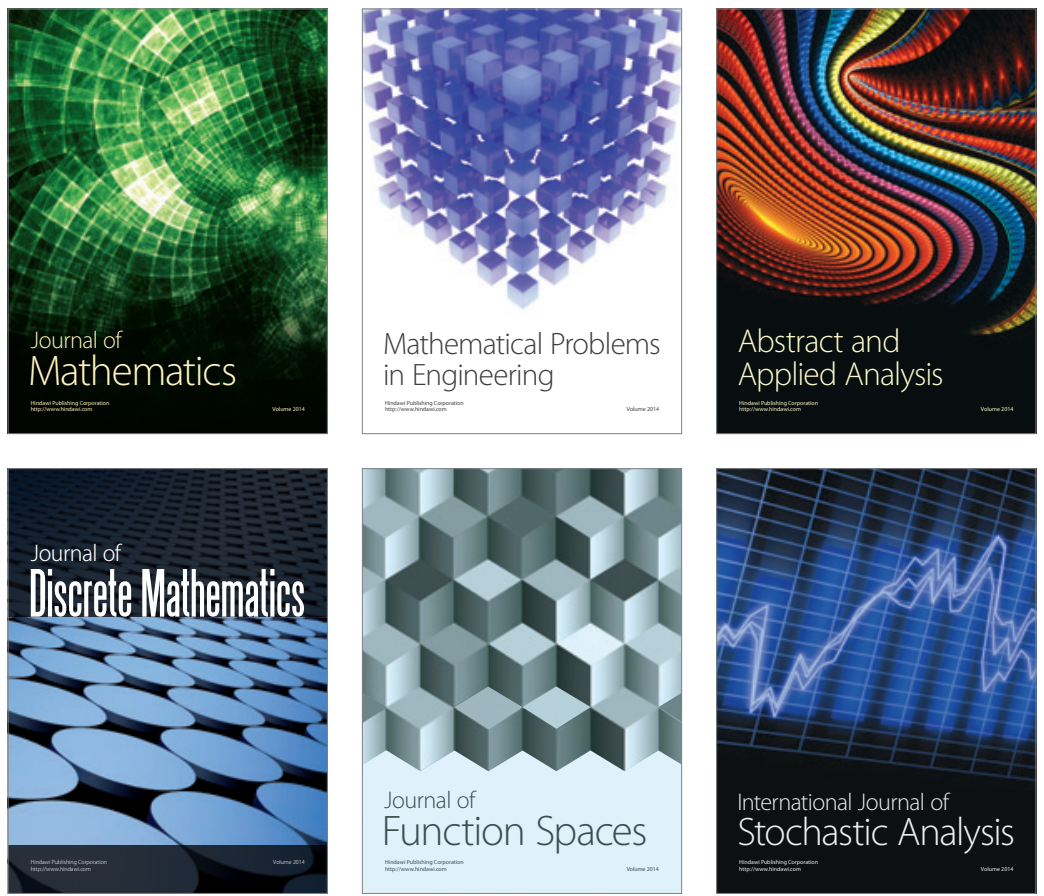

Journal of

Function Spaces

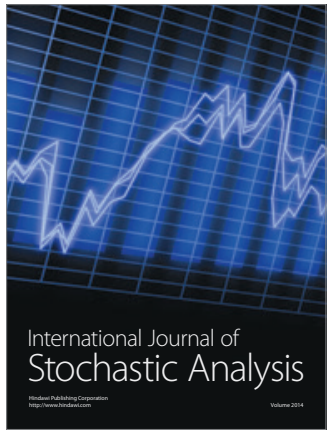

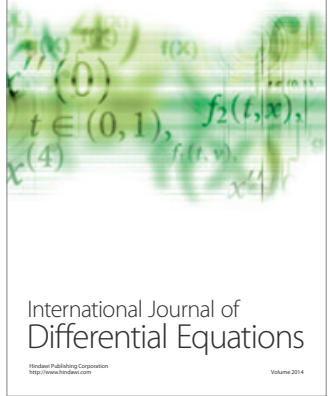
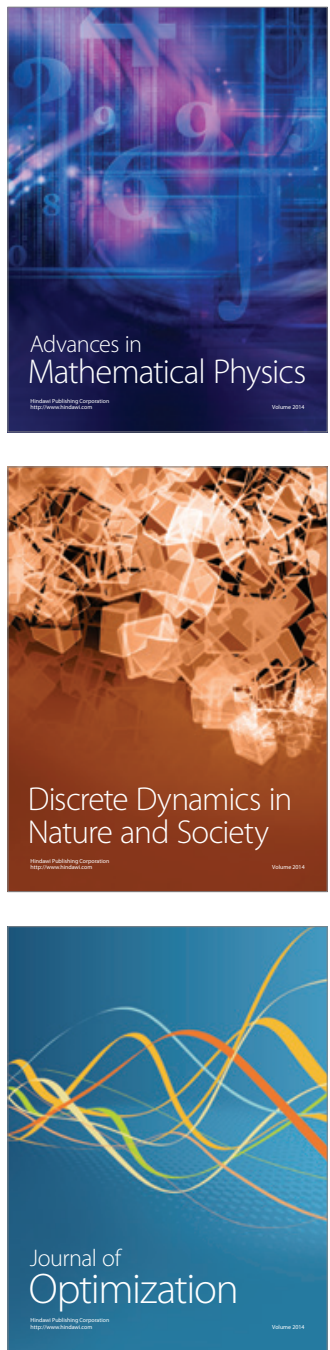